\title{
Uso de hábitat de Triaenodon obesus (Carcharhiniformes: Carcharhinidae), Rhincodon typus (Orectolobiformes: Rhincodontidae) y Manta birostris (Myliobatiformes: Myliobatidae) en el Parque Nacional Natural Gorgona, Pacífico colombiano
}

\author{
Paola Andrea Mejía-Falla ${ }^{1 *}$, Andrés Felipe Navia ${ }^{1}$, Rafael Lozano ${ }^{1}$, Alexander Tobón- \\ López $^{1}$, Krupskaya Narváez ${ }^{1}$, Luis Augusto Muñoz-Osorio ${ }^{1}$, Luz Marina Mejía-Ladino ${ }^{1}$ \& \\ Juliana López-García ${ }^{1}$ \\ 1. Fundación colombiana para la investigación y conservación de tiburones y rayas SQUALUS, Carrera 60A No 11-39, \\ Cali, Colombia; pmejia@squalus.org, anavia@squalus.org, rlozano@squalus.org, atobon@squalus.org, \\ knarvaez@squalus.org,lmunoz@squalus.org,1mejia@squalus.org,jlopez@squalus.org
}

Recibido 18-X-2013. Corregido 20-XI-2013. Aceptado 19-XII-2013.

\begin{abstract}
Habitat use by Triaenodon obesus (Carcharhiniformes: Carcharhinidae), Rhincodon typus (Orectolobiformes: Rhincodontidae) and Manta birostris (Myliobatiformes: Myliobatidae) in Gorgona Island National Natural Park, Colombian Pacific Ocean. The ecotouristic approach to sharks and rays has developed rapidly in the last 20 years. We studied three species in Gorgona Island from 427 dives ( 2004-2011), including 866 photographs and videos. There was at least one sighting in $34.4 \%$ of the dives, and $25.0 \%$ of the photos and videos were useful for identification of individuals. T. obesus was seen in all months of the year, and its presence was correlated with depths of $20-30 \mathrm{~m}$ (66.9\%), sandy-rocky bottoms (79.8\%), and western $(61.2 \%)$ and south (52.9\%) areas of the island. The southern area had groups of up to 9 individuals but abundance was not correlated with zone, depth, bottom type or temperature. $R$. typus and M. birostris were mainly seen at $28-33^{\circ} \mathrm{C}$, in the north, where plankton was more abundant. We identified individually five whale sharks, 15 manta rays and 38 whitetip sharks, 9 of which (one ray and 8 whitetip sharks) were recaptured in different months and years. Most of recaptured whitetip sharks were alone (63.06\%), resting $(49.37 \%)$ and on the bottom (82.91\%) in the same area of the photographic capture, suggesting a high philopatry of this species on the island; however, the recapture of three individuals in different zones indicate connectivity between of this species in the west, south and north zones. The Gorgona Island is an important area for breeding and growth of $T$. obesus, with presence of gravid females and births. It is also a seasonal passage area for $R$. typus and M. birostris, which occur most frequently between March and September, but do not form aggregations around the island. Rev. Biol. Trop. 62 (Suppl. 1): 329-342. Epub 2014 February 01.
\end{abstract}

Key words: elasmobranchs, photoidentification, population dynamics, phylopatry, Tropical Eastern Pacific.

En los últimos años, el auge de la industria ecoturistica a nivel mundial ha reforzado la idea que algunas especies son más valiosas vivas que muertas, especialmente aquellas con las cuales los seres humanos pueden interactuar (Cisneros-Montemayor, Barnes-Mauthe, AlAbdulrazzak, Navarro-Holm, Sumaila, 2013). Esta actividad de turismo con animales en vida silvestre se ha incrementado exponencialmente en los últimos 20 años (Higginbottom \& Scott, 2008; Clua, Burray, Legendre, Mourier
\& Planes, 2011; Gallagher \& Hammerschlag, 2011) y se promueve como una alternativa económica para la conservación de la naturaleza y de beneficios para las comunidades locales que reduce la presión sobre estos recursos en comparación al uso pesquero o deportivo (Topelko \& Dearden, 2005; de Lacy \& Whitmore, 2006; Gallagher \& Hammerschlag, 2011; CisnerosMontemayor et al., 2013). En cuanto a esta práctica con tiburones y rayas, se destacan aquellas realizadas con Rhincodon typus, 
Manta birostris y algunas especies arrecifales como Carcharhinus perezi, Gynglimostoma cirratum o Dasyatis americana, las cuales son la base de actividades turísticas como avistamiento, alimentación y buceo. Esta cercanía con la industria del turismo, ha facilitado el desarrollo de investigaciones sobre diversos temas como patrones de movimiento (Graham \& Roberts, 2007; Brooks, Rowart, Pierce, Jouannet \& Vely, 2010; Heupel, Simpfendorfer \& Fitzpatrick, 2010), estimaciones poblacionales (Meekan et al., 2006; Theberge \& Dearden, 2006; Holmberg, Norman \& Arzoumanian, 2008; Hussey, Stroh, Klaus, Chekchak \& Kessel, 2013) y estudios focales en especies como Orectolobus spp. (Huveneers, Luo, Otway \& Harcourt, 2009), Carcharias taurus (Bansemer \& Bennett, 2008, 2009, 2010), Stegostoma fasciatum (Dudgeon, Noad \& Lanyon, 2008) y Manta birostris (Luiz, Balboni, Kodja, Andrade \& Marum, 2009), entre otras.

La mayoría de estos estudios se han realizado en áreas geográficas como Australia (Meekan et al., 2006; Holmberg et al., 2009; Huveneers et al., 2009; Speed et al., 2011), Indonesia y Tailandia (Theberge \& Dearden, 2006; Stacey, Karam, Dwayer, Speed \& Meekan, 2008), el Mediterráneo (Canese et al., 2011), el Mar Rojo (Hussey et al., 2013), el Atlántico y el Caribe (Luiz et al., 2009; Cárdenas-Palomo, Herrera-Silveira \& Reyes, 2010; de la Parra-Venegas et al., 2011). Por su parte, en el Pacífico Oriental dichos trabajos se han iniciado recientemente y las publicaciones disponibles se restringen a poblaciones de Manta alfredi y M. birostris en Hawaii (Clark, 2010; Deakos, Bajer \& Bedjer, 2011) y Sphyrna lewi$n i$ en la isla Malpelo (Bessudo et al., 2011).

En la costa Pacífica colombiana, la mayoría de los estudios realizados con elasmobranquios han sido desarrollados a partir de datos de capturas de pesca, enfocándose por tanto, en aspectos taxonómicos y biológicos, siendo casi nulos los que vinculan a animales en su estado natural (Navia et al., 2009). En este sentido, información de animales in situ en costas colombianas, ha sido recopilada desde enero de 2003 a través de un Programa Nacional de
Avistamiento de Tiburones y Rayas (PNAT), el cual ha mostrado resultados importantes para diferentes áreas marinas protegidas de Colombia, en especial para el Parque Nacional Natural Gorgona (PNNG). Esta isla presenta una alta actividad de buceo recreativo, y en ella se han avistado individuos de especies críticamente amenazadas como Pristis perotteti, especies raras como Rhinobatos prahli, especies migratorias como $R$. typus y M. birostris, así como especies residentes como Triaenodon obesus (Mejía-Falla, Navia, Tobón \& Lozano, 2004; Navia, Narváez, Caicedo, Mejía-Falla \& Giraldo, 2005). El valor de este tipo de información colectada a través del tiempo y en colaboración con buzos recreativos ha sido resaltado en diferentes lugares del mundo (Ward-Paige \& Lotze, 2011).

Finalmente, la Isla Gorgona hace parte del Corredor Marino Este Tropical, por lo que podría presentar un importante rol en la dinámica poblacional de especies que han sido avistadas históricamente en la isla, como el aletiblanco ( $T$. obesus), el tiburón ballena ( $R$. typus) y la mantarraya (M. birostris). De esta forma, el objetivo de este trabajo fue evaluar el uso de hábitat que presentan estas especies alrededor de la isla, a partir de observaciones in situ y de la foto-identificación de individuos, técnica no invasiva que permite estudiar y censar animales en su medio natural.

\section{MATERIALES Y MÉTODOS}

Área de estudio: El Parque Nacional Natural Gorgona (PNNG) está conformado por una zona insular y un área marina circundante de $616.8 \mathrm{Km}^{2}$. La zona insular del PNNG está definida por la isla Gorgona y el islote de Gorgonilla. Esta área protegida está ubicada en el Pacífico colombiano, entre las coordenadas $2^{\circ} 55^{\prime} 45^{\prime \prime}-3^{\circ} 00^{\prime} 55^{\prime \prime}$ N y $78^{\circ} 09^{\prime} 00^{\prime \prime}$ 78¹4’30" W (Díaz, Pinzón, Perdomo, Barrios \& López-Victoria, 2001). Desde el punto de vista biogeográfico y ecológico, confluyen en sus aguas cinco tipos de comunidades tanto de hábitos costeros como oceánicos, y convergen especies propias del Pacífico Este, Central e 
Indopacífico, así como especies migratorias (Díaz et al., 2001).

Trabajo de campo y colecta de datos: Entre 2004 y 2011 se realizaron inmersiones con equipo SCUBA, en cuatro zonas de la isla (norte, sur, este y oeste) y en diferentes horas del día, para llevar a cabo censos visuales de T. obesus, R. typus y M. birostris. Se efectuó un censo por inmersión con un equipo de tres personas, registrando: fecha, zona, hora de inicio y fin de inmersión, temperatura de fondo y superficie, y profundidad mínima y máxima. Para cada individuo observado se determinó el sexo, estadío (cría, juvenil, adulto), ubicación (fondo, media agua, superficie), tipo de fondo (rocoso, arenoso, areno-rocoso, areno-coralino, cascajo y coralino), actividad (reposo, natación o alimentación), profundidad, temperatura y registro visual (foto, video o dibujo). Estos registros visuales se enfocaron en lugares corporales específicos de los individuos, teniendo en cuenta las siguientes características: $T$. obesus: aletas dorsales, aleta caudal y costados; $R$. typus: costados, área de las branquias, primera aleta dorsal y aleta caudal; M. birostris: dorso, vientre y aletas cefálicas.

Adicionalmente, a través del PNAT se recopilaron fotografías y videos de T. obesus, $R$. typus y $M$. birostris, tomadas por buzos recreativos durante el mismo periodo del estudio (2004 a 2011) en la isla Gorgona.

Análisis de datos: Datos de las inmersiones con y sin avistamiento fueron analizados mediante estadística descriptiva, incluyendo el porcentaje de avistamiento por zona (norte, sur, este y oeste), profundidad (intervalo 1: $<10 \mathrm{~m}$, intervalo 2: 10 a $19.9 \mathrm{~m}$, intervalo $3: 20$ a $29.9 \mathrm{~m}$, intervalo $4:>30 \mathrm{~m}$ ), temperatura del fondo (intervalo $1: 17$ a $21.9^{\circ} \mathrm{C}$, intervalo $2: 22$ a $26.9^{\circ} \mathrm{C}$, intervalo $3: 27$ a $31.9^{\circ} \mathrm{C}$ ), temperatura de superficie (intervalo 1: 23 a $27.9^{\circ} \mathrm{C}$, intervalo $2: 28$ a $32.9^{\circ} \mathrm{C}$ ) y tipo de fondo. Se realizó un análisis de correspondencia para evaluar la correlación entre las variables evaluadas y la presencia/ausencia de las tres especies en la Isla Gorgona.
Dado que el número de censos varió entre las zonas, y que en un mismo censo se pudo avistar una o dos especies de interés, los datos de los avistamientos se estandarizaron de la forma: número de inmersiones con avistamiento en la zona i / número de inmersiones totales en la zona $i^{*} 100$. Este valor correspondió al porcentaje de ocurrencia de las especies y sus valores totales no suman $100 \%$.

Para T. obesus, siendo la especie con mayor número de avistamientos, se realizaron pruebas no paramétricas de Kruskal-Wallis (H) y Mann-Whitney (U) entre el número de individuos avistados y cada una de las variables evaluadas; así mismo, se realizaron pruebas pos-hoc, donde fuese necesario, con el fin de evaluar diferencias en el número de individuos por cada variable.

Las fotografías y videos se analizaron inicialmente con Microsoft Office Picture Manager y Windows Media Player, respectivamente, con el fin de determinar si tenían la calidad necesaria para un análisis más detallado. Las fotos y los videos seleccionados se revisaron, teniendo en cuenta características propias y patrones de pigmentación específicos, muescas y anormalidades de los individuos. Cada foto o video de un individuo identificado, al cual se le asignó un nombre, se acompañó de un esquema con el contorno del individuo (lado izquierdo, lado derecho, aletas, dorso, vientre) con sus criterios de identificación enfatizados. Estas hojas incluyeron además los datos generales de la inmersión y de las fotos/videos tomados en las mismas.

A partir de las fotos y videos analizados, se definieron el número de individuos identificados y recapturados, así como el sexo y el estadio de cada uno de ellos. Análisis de los individuos identificados y recapturados incluyeron zonas, lugares y fechas de observación, así como las condiciones en las que fueron avistados.

\section{RESULTADOS}

Se efectuaron 427 inmersiones de buceo con tiempos entre 10 y 117 min (media 40.0; 
desv. estándar 11.17min), dependiendo del sitio de buceo y de la profundidad. La profundidad máxima de los censos varió entre 3.0 (zona este) y $45.0 \mathrm{~m}$ (zona oeste) $(20.5 ; 6.3 \mathrm{~m})$, la temperatura en el fondo entre 17.5 y $31.1^{\circ} \mathrm{C}(27.6$; $2.5^{\circ} \mathrm{C}$ ), y la temperatura superficial entre $23 \mathrm{y}$ $32.2{ }^{\circ} \mathrm{C}\left(27.9 ; 2.0^{\circ} \mathrm{C}\right)$.

Las censos se realizaron principalmente en la zona norte $(53.4 \%)$, seguida por la este $(22.7 \%)$ y oeste $(19.9 \%)$. El $34.4 \%$ de las inmersiones presentaron avistamiento de al menos una de las tres especies objetivo. La zona este mostró la probabilidad de avistamiento más baja (1.0\%) mientras que las zonas oeste y sur, con menor número de inmersiones, tuvieron mayores probabilidades de avistamiento (61.2 y 52.9\%, respectivamente; Fig. 1).

Considerando el número de inmersiones por zona, se encontró que $R$. typus y $M$. birostris se avistaron principalmente en la zona norte de la isla (3.1 y 10.1\% respectivamente) y T. obesus fue observado con mayor frecuencia en las zonas oeste $(61.2 \%)$ y sur $(52.9 \%)$ (Cuadro 1). Los tiburones ballena se avistaron entre los meses de abril y agosto y se observaron en la superficie, solitarios, a no más de $10 \mathrm{~m}$ de profundidad, y a temperaturas entre 28 y $33^{\circ} \mathrm{C}$ $(100 \%)$. Las mantarrayas se observaron entre marzo y septiembre, principalmente a media agua y a las mismas temperaturas del tiburón ballena $(66.7 \%)$, siendo avistados entre uno y tres individuos. Todos los individuos de estas dos especies fueron observados en natación lenta $(100 \%)$.

El tiburón aletiblanco se observó en todos los meses del año, principalmente entre los 20 y $30 \mathrm{~m}(66.9 \%)$, temperaturas entre los 27 y

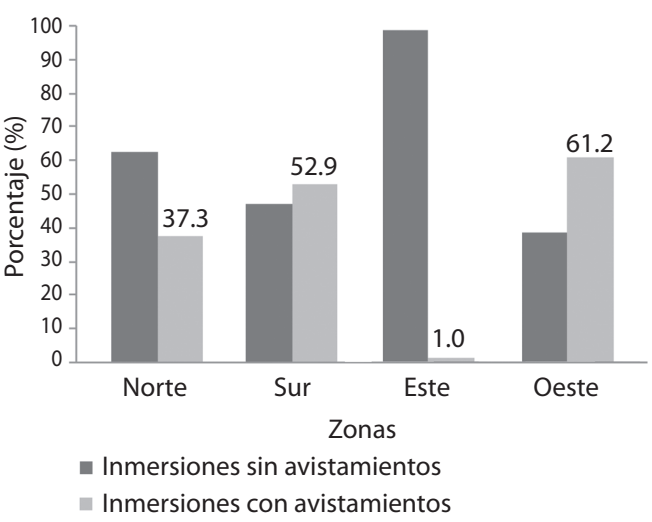

Fig. 1. Porcentaje de inmersiones con y sin avistamiento por zona en la Isla Gorgona.

Fig. 1. Percentage of dives with and without sighting by zone in Gorgona Island.

$\operatorname{los} 32^{\circ} \mathrm{C}(93.3 \%)$ y en fondos areno-rocosos (79.8\%) (Cuadro 2). Así mismo, la mayoría de los aletiblancos avistados, se observaron solitarios $(63.1 \%)$, en reposo $(49.4 \%)$ y en el fondo $(82.9 \%)$; siendo frecuente encontrarlos posados, con las aletas pectorales extendidas y con la aleta anal y caudal (lóbulo ventral) tocando levemente el fondo.

El análisis de correspondencia indicó que la presencia de T. obesus está relacionada principalmente a la zona sur y oeste, a fondos areno-rocosos y temperaturas entre 27 y $32^{\circ} \mathrm{C}$. Por su parte, la presencia de $M$. birostris y $R$. typus se correlacionó solo con la zona norte (Fig. 2).

Específicamente para T. obesus, las mayores congregaciones (mayores a siete individuos) se avistaron en la zona sur, en especial en el sitio conocido como la Cazuela, a profundidades mayores a $27 \mathrm{~m}$; en esta misma

\section{CUADRO 1}

Porcentaje de ocurrencia de T. obesus, R. typus y M. birostris por zona de buceo en la Isla Gorgona

TABLE1

Percentage of occurrence of T. obesus, R. typus and M. birostris by diving zone in Gorgona Island

\begin{tabular}{cccccc} 
Variable & Categorías & No. total inmersiones & Triaenodon obesus & Rhincodon typus & Manta birostris \\
\multirow{3}{*}{ Zona } & Norte & 228 & 28.5 & 3.1 & 10.1 \\
& Oeste & 85 & 61.2 & 0.0 & 0.0 \\
& Este & 97 & 0.0 & 1.0 & 0.0 \\
& Sur & 17 & 52.9 & 0.0 & 5.9 \\
\hline
\end{tabular}




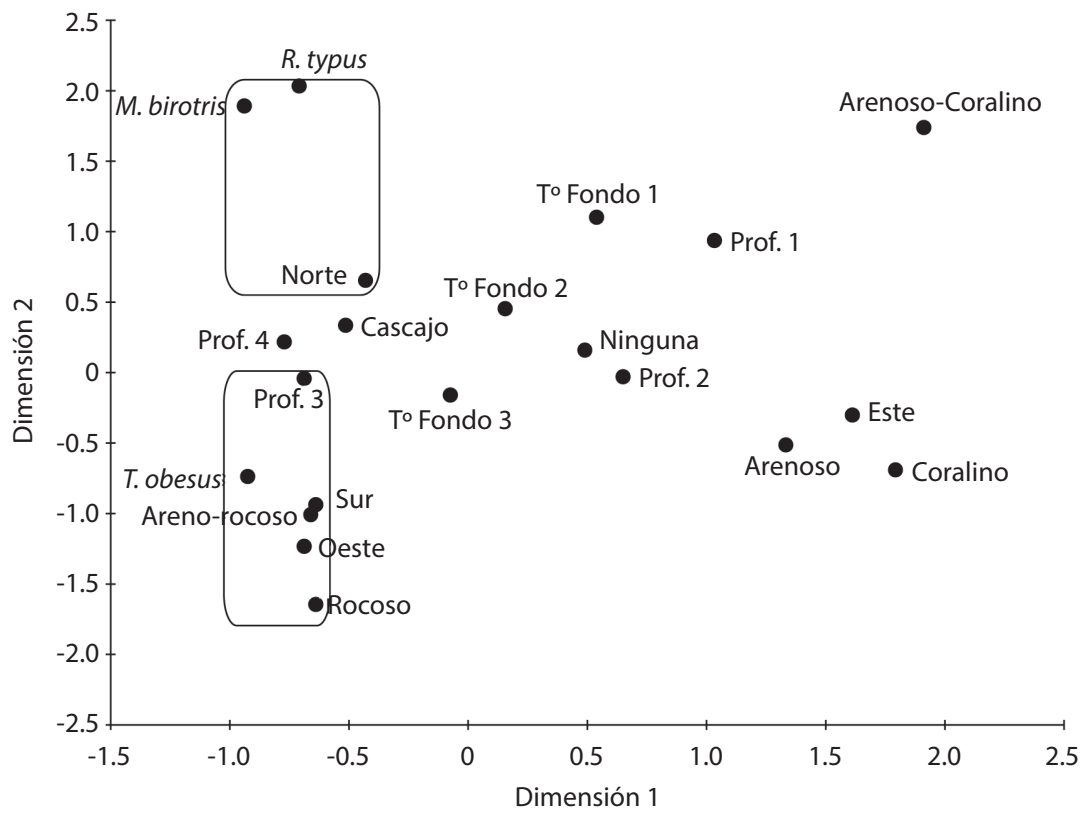

Fig. 2. Análisis de correspondencia de la presencia/ausencia de T. obesus, R. typus y M. birostris con las variables del hábitat (zona, tipo y temperatura del fondo). Las características de fondo sólo fueron consideradas para T. obesus. Dimensión 1: Eigenvalue 0.50, Inercia 15.62\%; Dimensión 2: Eigenvalue 0.30, inercia 9.37\%.

Fig. 2. Correspondence analysis of presence/absence of T. obesus, R. typus and M. birostris with the habitat variables (zone, type and temperature of the bottom). The bottom characteristics were only considered for T. obesus. Dimension 1: Eigenvalue 0.50, Inertia 15.62\%; Dimension 2: Eigenvalue 0.30, Inertia 9.37\%.

\section{CUADRO 2}

Porcentaje de ocurrencia de T. obesus por profundidad máxima, tipo y temperatura del fondo en la Isla Gorgona

\section{TABLE 2}

Percentage of occurrence of $T$. obesus by maximum depth, bottom type and temperature in Gorgona Island

\begin{tabular}{lcc}
\multicolumn{1}{c}{ Variable } & Categorías & $\%$ \\
Profundidad máxima & $<10 \mathrm{~m}$ & 0.0 \\
& $10-19.9 \mathrm{~m}$ & 19.4 \\
& $20-29.9 \mathrm{~m}$ & 66.9 \\
Fondo & $>30 \mathrm{~m}$ & 13.7 \\
& Areno-rocoso & 79.8 \\
& Arenoso & 7.9 \\
Temperatura de fondo & Cascajo & 2.6 \\
& Rocoso & 9.7 \\
& $17-21.9$ & 0.0 \\
& $22-26.9$ & 6.7 \\
& $27-31.9$ & 93.3 \\
\hline
\end{tabular}

zona, observaciones de hasta cuatro individuos fueron bajas (Fig. 3). En la zona norte se observaron principalmente entre uno y tres individuos, aunque en La Tiburonera se observaron grupos de seis y siete individuos a profundidades mayores a $23 \mathrm{~m}$ (Fig. 3). Sin embargo, no se encontraron diferencias significativas en la abundancia de T. obesus entre las zonas, la profundidad, el tipo y la temperatura del fondo (Cuadro 3).

Se tomaron y recopilaron 684 fotografías y 182 videos, de los cuales el 75\% correspondieron a $T$. obesus, $19 \%$ a $M$. birostris y $6 \%$ a $R$. typus. El $25 \%$ de los registros visuales fueron útiles para la diferenciación de individuos; a partir de ellos, se identificaron 38 aletiblancos, 15 mantarrayas y cinco tiburones ballena (Cuadro 4). Un total de nueve individuos (una $M$. 


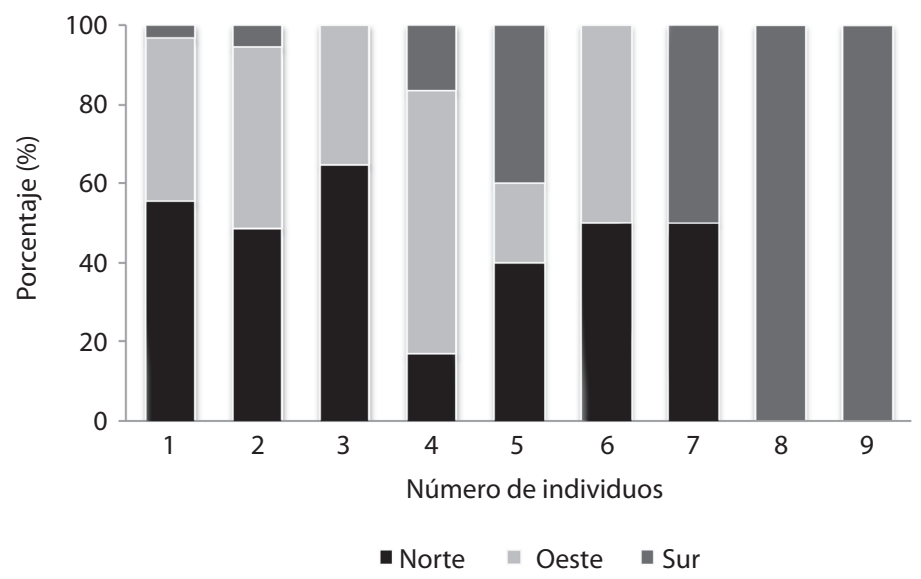

Fig. 3. Porcentaje de la abundancia (número de individuos por avistamiento) de T. obesus por zona de buceo.

Fig. 3. Percentage of abundance (number of individuals per sighting) of $T$. obesus by diving zone.

\section{CUADRO 3}

Valores de las pruebas estadísticas de la diferencia de la abundancia de T. obesus (número de individuos por avistamiento) con la zona, profundidad máxima, tipo y temperatura de fondo

\section{TABLE 3}

Statistical test values of the difference in the abundance of $T$. obesus (number of individuals per sighting) with the zone, maximum depth, bottom type and temperature

\begin{tabular}{lccc}
\multicolumn{1}{c}{ Variable } & $\mathrm{n}$ & Estadístico & $\mathrm{p}$ \\
Zona & 126 & $\mathrm{H}=5.597$ & 0.061 \\
Tipo de fondo & 114 & $\mathrm{H}=3.772$ & 0.287 \\
Profundidad & 124 & $\mathrm{H}=1.948$ & 0.378 \\
Temperatura de fondo & 111 & $\mathrm{U}=0.927$ & 0.354 \\
\hline
\end{tabular}

birostris y ocho T. obesus) fueron reavistados, en meses, e incluso años diferentes; de $R$. typus no se obtuvieron recapturas. La mantarraya ("Quinto") correspondió a un macho adulto capturado fotográficamente en mayo de 2004 y recapturado en julio de 2006 (Fig. 4).

Los aletiblancos reavistados correspondieron a tres machos ("Puntudo", "Adán" y "Cupido") y a cinco hembras ("Afrodita", "Delfina", "Medialuna", "Pecosa" y "Scuba"). Todos, excepto "Pecosa" correspondieron a individuos adultos. Los lugares y fechas de marca y recaptura fotográfica de cada individuo se indican en el Cuadro 5. "Afrodita" fue el individuo con

\section{CUADRO 4}

Número de registros fotográficos y fílmicos analizados y usados para la identificación (ID) de individuos de T. obesus, $R$. typus y M. birostris, y número de animales identificados por sexo (H: Hembras, M: machos, I: indeterminado)

TABLE 4

Number of photographs and videos analyzed and used for identification (ID) of individuals of T. obesus, $R$. typus and M. birostris, and the number of identified animals by sex (H: Female, M: male, I: indeterminate)

\begin{tabular}{|c|c|c|c|c|c|c|c|}
\hline Especie & Registros Fotográficos & Registros Fílmicos & Usados para ID & Animales ID & $\mathrm{H}$ & M & I \\
\hline Triaenodon obesus & 538 & 111 & 164 & 38 & 20 & 17 & 1 \\
\hline Manta birostris & 116 & 51 & 46 & 15 & 6 & 7 & 2 \\
\hline Rhincodon typus & 30 & 20 & 8 & 5 & 0 & 3 & 2 \\
\hline Total & 684 & 182 & 218 & 58 & 29 & 24 & 5 \\
\hline
\end{tabular}



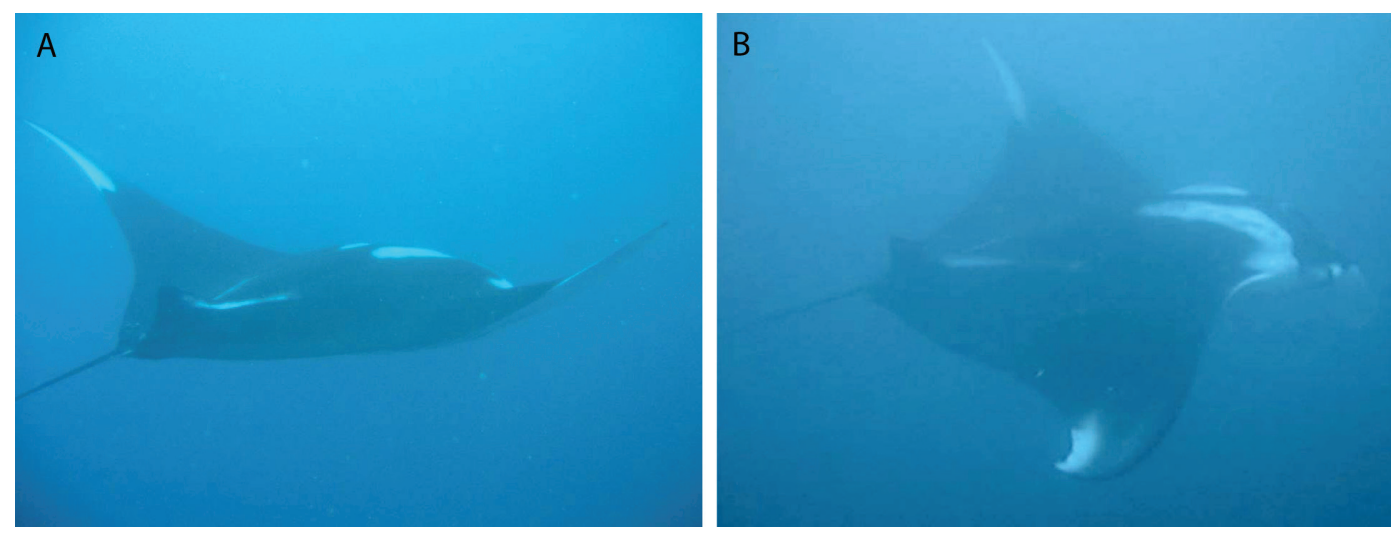

Fig. 4. Macho adulto de mantarraya ("Quinto") observado en (A) mayo 2004 y (B) julio 2006.

Fig. 4. Adult male of manta ray ("Quinto") observed in (A) May 2004 and (B) July 2006.

\section{CUADRO 5}

Individuos de T. obesus identificados en la Isla Gorgona, indicando las zonas (N: Norte; S: Sur; W: Oeste; E: Este), lugares y fechas de captura y recaptura

TABLE 5

Individuals of T. obesus identified in Gorgona Island, indicating the zones

(N: North, S: South, W: West, E: East), places and dates of capture and recapture

\begin{tabular}{|c|c|c|c|}
\hline $\begin{array}{l}\text { Individuo } \\
\text { identificado }\end{array}$ & Sexo & $\begin{array}{l}\text { Zona, lugar y fecha de } \\
\text { captura fotográfica }\end{array}$ & Zona, lugar y fecha de recaptura fotográfica. \\
\hline Afrodita & $\mathrm{H}$ & W-Montañita II-Setiembre, 2004 & $\begin{array}{l}\text { W- Montañita II-Julio y Setiembre, } 2005 \\
\text { S-Cazuela-Junio, } 2011 .\end{array}$ \\
\hline Delfina & $\mathrm{H}$ & W- Montañita II-Mayo 17, 2011 & $\begin{array}{l}\text { W-Montañita II-Mayo, 19, 2011; } \\
\text { Junio, } 5 \text { y 7, 2011; Setiembre, } 2011 .\end{array}$ \\
\hline Medialuna & $\mathrm{H}$ & S-Cazuela- Agosto, 2008 & S-Cazuela-Junio, 2011. \\
\hline Pecosa & $\mathrm{H}$ & W-Montañita II-Agosto, 2007 & $\begin{array}{l}\text { N-Tiburonera-Setiembre, 2007; Octubre, 2007; } \\
\text { Noviembre } 4 \text { y } 25,2007 .\end{array}$ \\
\hline Scuba & $\mathrm{H}$ & W-Montañita II-Agosto, 2010 & $\begin{array}{l}\text { W-Montañita II-Noviembre, 2010; Mayo } 17 \text { y 19, 2011; } \\
\text { Setiembre, } 2011 .\end{array}$ \\
\hline Puntudo & M & W-Montañita II-Agosto, 2008 & S-Cazuela-Junio, 2011. \\
\hline Adán & M & W-Montañita II-Setiembre, 2006 & W-Montañita II-Octubre, 2006; Agosto, 2007. \\
\hline Cupido & M & W-Montañita II-Junio, 2010 & W-Montañita II-Agosto, 2010. \\
\hline
\end{tabular}

mayor número de recapturas en años diferentes, siendo capturada fotográficamente por primera vez en septiembre de 2004 en Montañita II (zona oeste) y recapturada en junio y septiembre de 2005 en la misma zona y en junio de 2011 en la Cazuela (zona sur); esta hembra fue observada siempre en estado de preñez (Fig. 5A, B, C). "Delfina, Pecosa y Scuba" fueron recapturados cuatro veces; los dos primeros en
2007, y "Scuba" en 2010 y 2011 (Fig. 5D-G). "Pecosa" fue capturada en Montañita II en agosto 2007 y recapturada en los siguientes tres meses en la Tiburonera (zona norte), donde fue observada siempre en el mismo lugar (Fig. 5H, I). "Puntudo" fue capturado en Montañita II en agosto 2008 y recapturado 3 años después en la Cazuela. Un caso particular correspondió a un macho (Cupido) que siempre fue observado 

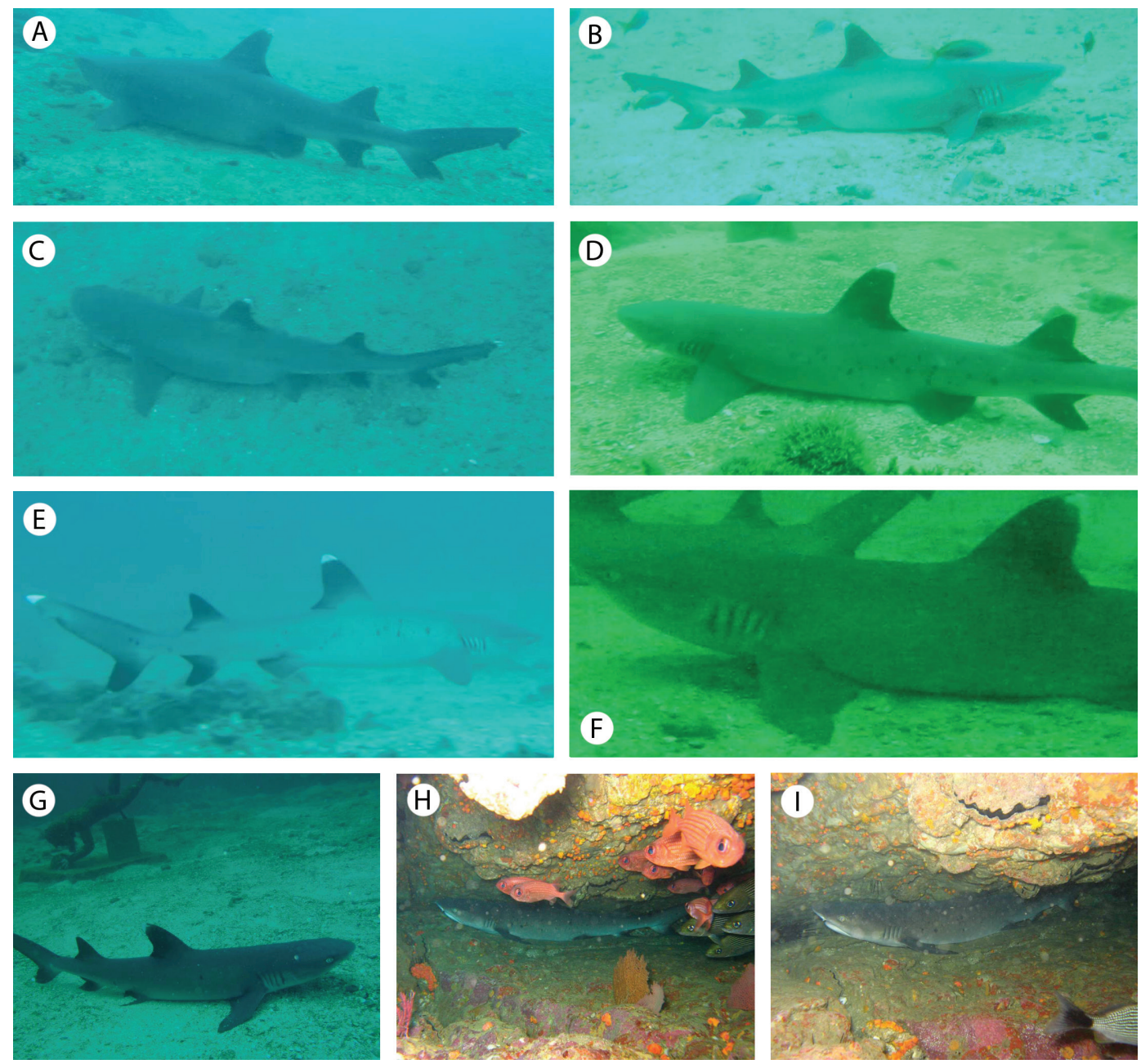

Fig. 5. Hembras de T. obesus avistadas y recapturadas en la Isla Gorgona, entre los años 2004 y 2011. "Afrodita": hembra avistada en (A) septiembre 2004, y (B) julio 2005, (C) junio 2011. "Delfina": hembra avistada en Montañita II en (D) mayo y (E) septiembre 2011. "Scuba": hembra avistada en Montañita II en (F) agosto 2010 y (G) septiembre 2011. "Pecosa": hembra avistada en (H) septiembre y (I) noviembre 2007 en Montañita II.

Fig. 5. Female T. obesus sighted and recaptured in Gorgona Island, between 2004 and 2011. "Afrodita": female sighted in (A) September 2004, (B) July 2005, and (C) June 2011. "Delfina": female sighted in Montanita II in (D) May and (E) September 2011. "Scuba": female sighted in Montañita II in (F) August 2010 and (G) September 2011. "Pecosa": female sighted in (H) September and (I) November 2007 in Montañita II.

junto a una hembra (con "Alik" en junio de 2010 y con "Scuba" en agosto del mismo año), siempre en el mismo lugar (Montañita II).

\section{DISCUSIÓN}

El Parque Nacional Natural Gorgona es una de las zonas marinas del Pacífico colombiano con mayor número de especies de tiburones y rayas registrados. Así mismo, la mayoría de estas especies presentan individuos maduros, neonatos y juveniles, lo que convierte a esta Isla en un área prioritaria para la conservación de estas especies en la región (Mejía-Falla et al., 2007; Hleap, Lozano, Navia \& Payán, 2010). 
En la zona este, donde sólo se observó al tiburón ballena, se han registrado especialmente especies de rayas de hábitos bentónicos como Urobatis halleri, Dasyatis longa y Narcine entemedor y pelágicas como Mobula thurstoni (Fundación Squalus, in prep.). La presencia de fondos arenosos y coralinos, así como la ausencia de aletiblancos u otros depredadores potenciales como Carcharhinus limbatus, pueden explicar la mayor frecuencia de observación de dichas especies en esta zona de la Isla.

Las zonas oeste y sur de la Isla parecen tener mayor importancia en la dinámica local de $T$. obesus, dado que en éstas se observaron de manera regular grupos más grandes, incluso con individuos en diferentes estados de desarrollo, incluyendo hembras grávidas. Esto sugiere que estas dos zonas concentran la mayor cantidad de aletiblancos de la isla y que en ellas se produciría el mayor aporte de crías a la población. Lo anterior, junto con el registro de un posible alumbramiento en el mes de noviembre en la zona norte de la isla (Mejía-Falla, Tobón-López, Navia, Narváez \& Lozano, 2010), soportan la importancia de Gorgona en los procesos vitales de esta especie.

Las observaciones de hembras grávidas de T. obesus, entre junio y noviembre, sugieren que la época de alumbramiento se encuentra en el segundo semestre del año, específicamente entre octubre y noviembre. Sin embargo, estas fechas no concuerdan con los trabajos de Shaller (2006), Robbins (2006) y Whitney, Pyle, Holland \& Barcz, (2012) quienes sugieren que los nacimientos ocurren entre Mayo y Junio. Estas diferencias pueden ser dadas por variaciones poblacionales, siendo dichos trabajos realizados en áreas templadas.

Las observaciones de la hembra "Afrodita", con recapturas fotográficas en tres años diferentes (septiembre de 2004, junio y septiembre de 2005, junio de 2011), y siempre en estado de gravidez, permiten inferir un periodo de gestación de alrededor de un año. Este periodo tentativo de reproducción concuerda con lo reportado por Shaller (2006) y Robbins (2006) quienes registran un periodo de gestación entre
12-13 meses. Estos mismos autores plantean que las hembras están listas para aparearse 95 días después del parto, tienen un solo evento reproductivo bianual y fecundidad promedio baja (1.6 a 2.1 crías por camada).

Basados en la talla de madurez $(82-116 \mathrm{~cm}$ Longitud Total [Lt] para machos y $102-122 \mathrm{~cm}$ Lt para hembras) y la talla de nacimiento (43$60 \mathrm{~cm} \mathrm{Lt}$ ) propuestas para la especie (Randall, 1977; Robbins, 2006; Shaller, 2006; Whitney et al., 2012), y en los estadíos de los ejemplares avistados en el estudio (estimados visualmente), se plantea que en las zonas evaluadas de la Isla Gorgona, se encuentran individuos juveniles y adultos, reproductivamente activos, pero no así, neonatos. De esta forma, se sugiere que al ser las zonas evaluadas altamente frecuentadas por buzos recreativos, los neonatos podrían estar usando otras áreas menos impactadas por esta actividad.

Diferentes autores han planteado alta fidelidad del aletiblanco a los lugares de asentamiento y agregación (Musick, Harbin \& Compagno, 2004; Speed et al., 2011; Whitney et al., 2012). En particular, Speed et al. (2011) sugieren que esta fidelidad se presenta a través del tiempo, e incluso en grandes sistemas arrecifales continuos, proponiendo que ello se debe principalmente a eventos reproductivos, más que a facilidades de alimento o protección contra depredadores. Así mismo, Whitney et al. (2012) registraron alta filopatría de la especie con múltiples recapturas fotográficas de un mismo ejemplar en un mismo sitio hasta por siete años, y documentando mayor filopatría en las hembras que en los machos. Nuestros resultados concuerdan, en parte, con los trabajos expuestos, donde algunos individuos identificados fueron recapturados fotográficamente en la misma área, en meses y años diferentes (e.g. "Medialuna", "Scuba", "Adán"). Sin embargo, tres individuos fueron observados en lugares diferentes de la isla, en años distantes ("Afrodita" y "Puntudo") e incluso en un mismo año ("Pecosa"). A pesar de los movimientos entre las zonas de la isla, se considera que, en general, el aletiblanco presenta filopatría a sus lugares de asentamiento y agregación, en especial en la 
Cazuela (zona sur) y Montañita II (zona oeste). Así mismo, observaciones del macho adulto "Cupido" avistado con hembras diferentes en meses cercanos y la observación de hembras preñadas en diferentes años (e.g. "Afrodita") sugieren que la filopatría en estas zonas puede estar ligada a eventos reproductivos.

Con respecto al tiburón ballena, resultados e información previa de la especie en la zona de estudio, muestran una presencia estacional con dominancia entre los meses de marzo y septiembre (Mejía-Falla et al., 2004; Navia et al., 2005). Así mismo, tanto el número de registros como la frecuencia de los mismos, sugieren que el número de individuos que frecuentan la isla es muy bajo en comparación con las frecuencias de avistamiento en otras áreas geográficas, como el Atlántico brasilero (Hazin, VaskeJunior, Oliveira, Macena, \& Carvalho, 2008), el Océano Índico oeste (Brooks et al., 2010) y en especial, el Caribe mexicano (de la ParraVenegas et al., 2011). Al respecto, Arnborn \& Papastravrou (1988) y Cárdenas-Palomo et al. (2010) sugieren que la estacionalidad y las mayores agregaciones de tiburón ballena en las islas Galápagos y el Caribe mexicano están asociadas a una mayor disponibilidad de zooplancton, específicamente ictioplancton (de la Parra-Venegas et al., 2011). Por su parte, Heyman, Graham, Kjerfve \& Johannes (2001) y Wilson, Taylor \& Pearce (2001) notaron mayores abundancias de tiburón ballena durante eventos de "La Niña" en aguas australianas, relacionándolas así con la temperatura superficial del mar. En este sentido, Arnborn \& Papastravrou (1988) y Cárdenas-Palomo et al. (2010) registraron mayor presencia de la especie en temperaturas superficiales entre $23.5^{\circ} \mathrm{C}$ y $30.0^{\circ} \mathrm{C}$ en las islas Galápagos y en el Caribe Mexicano, mientras nuestro estudio indica mayor frecuencia en temperaturas más altas $\left(28\right.$ y $\left.33^{\circ} \mathrm{C}\right)$.

En este estudio, la mayor parte de los avistamientos de tiburón ballena fueron registrados en superficie y a profundidades inferiores a los $10 \mathrm{~m}$. Esta característica es similar a la reportada por Eckert \& Stewart (2001) quienes registraron $90 \%$ de sus avistamientos en aguas $<20 \mathrm{~m}$, atribuyendo este fenómeno, nuevamente, a que en las capas menos profundas se encuentran las mayores concentraciones del zooplancton. Aunque Colman (1997) y Graham \& Roberts (2007) sugieren segregación por tallas y sexos para el tiburón ballena en los arrecifes de Australia y Belice, el bajo número de animales fotoidentificados en este trabajo no permite estimar con precisión si esta característica se presenta en isla Gorgona. La estacionalidad de la especie y la ausencia de grandes concentraciones de $R$. typus en el Pacífico colombiano, sugieren que los animales avistados en esta zona se estarían desplazando entre sitios de agregación o concentración adyacentes, como podrían ser islas Galápagos y el Pacífico mexicano (Enríquez-Andrade et al., 2003).

Con respecto a las mantarrayas, aunque tradicionalmente se han identificado como Manta birostris, es necesario desarrollar estudios que permitan confirmar la identidad taxonómica de los ejemplares que se avistan en la isla, ya que recientemente otra especie, Manta alfredi, ha sido reconocida como válida (Marshall, Compagno \& Bennett, 2009). Aunque la distribución conocida de $M$. alfredi se concentra en el Pacífico central, Pacífico occidental e Indo Pacífico (e.g. Anderson, Adam \& Goes, 2011; Couturier et al., 2011; Marshall, Dudgeon \& Bennett, 2011) no es descartable que pueda estar presente en la islas del Pacífico Oriental Tropical (POT), pues ya ha sido registrada en grandes grupos en el Archipiélago de Hawaii (Clark, 2010).

Aunque el número de individuos identificados de mantas (15) fue mayor que los del tiburón ballena (cinco), se sugiere que el número total de individuos de $M$. birostris que frecuentan la isla es igualmente reducido. En general, grandes concentraciones de esta especie no han sido registradas en otras localidades; por ejemplo, Clark (2010) documentó la presencia de tan solo 29 ejemplares de $M$. birostris en Hawaii durante 15 años. Por el contrario, para $M$. alfredi, Marshall et al. (2011) registraron un grupo de 449 individuos en Mozambique, mientras que Deakos et al. (2011) reportaron 209 individuos en Hawaii y 
Couturier et al. (2011) identificaron 388 en una sola isla en Australia. En este sentido, y dado que Mejía-Falla \& Navia (2011) identificaron a $M$. birostris como una especie propensa a extinción local en el POT, y que dicha probabilidad podría incrementarse si los grupos poblacionales son de tamaños reducidos, se considera prioritario continuar desarrollando estudios más detallados sobre la abundancia de esta especie en esta región biogeográfica.

La gran diferencia entre el número de individuos observados de $M$. birostris y $M$. alfredi en sus rangos de distribución, podría deberse a los hábitos de las especies: M. birostris es de hábitos oceánicos y migratorios (Dewar et al., 2008; Deakos, 2010) mientras que $M$. alfredi es una especie costera, con incluso indicios significativos de fidelidad al sitio (Deakos et al., 2011; Marshall et al., 2011). El patrón estacional de $M$. birostris ha sido registrado en Brasil (Luiz et al., 2009), Hawaii (Clark, 2010) y el Pacífico colombiano (Mejía-Falla et al., 2004; Navia et al., 2005). En este aspecto, Freedman \& Roi (2012) sugieren que las migraciones se correlacionan con la temperatura superficial del mar, en especial, aguas cálidas y altas concentraciones de clorofila, lo que se traduce en una mayor disponibilidad de alimento. Al igual que con el tiburón ballena, la explicación más aceptada sobre la estacionalidad de $M$. birostris, ha sido la relacionada con los eventos alimentarios (Luiz et al., 2009; Clark, 2010). De manera similar para M. alfredi, Anderson et al. (2011) registran que la abundancia de la especie en las Islas Maldivas está fuertemente influenciada por los patrones estacionales de los vientos monzónicos que favorecen los afloramientos de fitoplancton; y Couturier et al. (2011) sugieren que sus migraciones en Australia obedecen a la temperatura del agua, corrientes y disponibilidad de alimento.

En este aspecto, y específicamente para el área de estudio, se han documentado mayores abundancias de zooplancton entre los meses de febrero y abril, con dominancia de copépodos y quetognatos, y baja proporción de larvas de peces (Giraldo, Rodríguez-Rubio \& Zapata, 2008). Dado que estos meses no coinciden con el periodo estacional de $R$. typus y $M$. birostris en la Isla Gorgona, se sugiere que la disponibilidad de alimento (en cantidad o composición) no es óptima para estas dos especies, limitando así, la presencia de grandes agregaciones de las mismas. Sin embargo, la mayor presencia de estas dos especies en la zona norte de la isla, si puede estar relacionada con una mayor abundancia de larvas de peces presentes en dicha zona (Giraldo \& Zapata, 2006; Escarria, Beltrán-León, Giraldo \& Zapata, 2007).

Los resultados de este estudio, con respecto al tiburón ballena y la mantarraya necesitan fortalecerse, en especial porque las probabilidades de recaptura fotográfica de especies estacionales son muy bajas, tendiendo a subestimar el número de individuos, tal como ha sido registrado en otros estudios que utilizan la fotoidentificación como técnica de conteo poblacional (Graham \& Roberts, 2007). En ese sentido, es importante fomentar la participación de los buzos recreativos en estas investigaciones, dado que los aportes de sus fotografías y videos pueden incrementar considerablemente el número de animales registrados y la frecuencia con que se avistan en un área específica, como la isla Gorgona. Sin embargo, se recomienda que las actividades de buceo recreativo, entre los meses de octubre y diciembre en las zonas sur y occidental de la Isla, estén bajo el acompañamiento de funcionarios del Parque, dada la importancia de estas zonas y meses para la reproducción de $T$. obesus.

\section{AGRADECIMIENTOS}

Los autores agradecen al Fondo para la Acción Ambiental, Conservación Internacional, PADI Foundation y la Iniciativa para Especies Amenazadas por la co-financiación de este proyecto. A Embarcaciones Asturias y al personal del Parque Nacional Natural Gorgona por su apoyo en el trabajo de campo. A todos los buzos y colaboradores del Programa Nacional de Avistamiento de tiburones y rayas (PNAT) por las fotos, videos y los reportes de avistamiento de tiburones y rayas. Este proyecto se 
realizó bajo el permiso de investigación PIBDDTSO 011-10.

\section{RESUMEN}

Se evaluó el uso de hábitat del aletiblanco Triaenodon obesus, la mantaraya Manta birostris y el tiburón ballena Rhincodon typus en la Isla Gorgona, a partir de 427 inmersiones de buceo y del análisis de 866 fotografías y videos. T. obesus se observó a lo largo del año, y su presencia se correlacionó con profundidades entre 20 y 30m (66.9\%), fondos areno-rocosos $(79.8 \%)$ y las zonas occidental $(61.2 \%)$ y sur $(52.9 \%)$, con mayores congregaciones en esta última zona. $R$. typus y $M$. birostris se avistaron entre 28 y $33^{\circ} \mathrm{C}$ y su presencia se correlacionó con la zona norte de la isla, área de mayor abundancia de zoo e ictioplancton. Se identificaron 5 tiburones ballena, 15 mantarrayas y 38 aletiblancos, 9 de los cuales fueron recapturados en años diferentes. La mayoría de los aletiblancos recapturados fueron observados solos, en reposo y sobre el fondo y en la misma zona, sugiriendo alta filopatría; sin embargo, la recaptura de tres individuos en zonas diferentes, indican conectividad de la especie en la isla. Gorgona es un área importante para la reproducción y crecimiento del aletiblanco, y un área de paso para el tiburón ballena y la mantarraya, los cuales se observan con mayor frecuencia entre marzo y septiembre, pero no forman agregaciones.

Palabras clave: elasmobranquios, fotoidentificación, dinámica poblacional, filopatría, Pacífico Oriental Tropical.

\section{REFERENCIAS}

Anderson, R. C., Adam, M. S. \& Goes, J. I. (2011). From monsoons to mantas: seasonal distribution of Manta alfredi in the Maldives. Fisheries Oceanography, 20: 104-113.

Arnborn, T. \& Papastravrou, V. (1988). Fish in association with whale sharks Rhiniodon typus near the Galapagos islands. Noticias de Galapagos, 46: 13-15.

Bansemer, C. S. \& Bennett, M. B. (2008). Multi-year validation of photographic identification of grey nurse sharks, Carcharias taurus, and applications for non-invasive conservation research. Marine and Freshwater Research, 59: 322-331.

Bansemer, C. S. \& Bennett, M. B. (2009). Reproductive periodicity, localised movements and behavioural segregation of pregnant Carcharias taurus at Wolf Rock, southeast Queensland, Australia. Marine Ecology Progress Series, 374: 215-227.

Bansemer, C. S. \& Bennett, M. B. (2010). Retained fishing gear and associated injuries in the east Australian grey nurse sharks (Carcharias taurus): implications for population recovery. Marine and Freshwater Research,61: 97-103.
Bessudo, S., Soler, G., Klimley, A. P., Ketchum, J. T., Hearn, A. \& Arauz, R. (2011). Residency of the scalloped hammerhead shark (Sphyrna lewini) at Malpelo Island and evidence of migration to other islands in the Eastern Tropical Pacific. Environmental Biology of Fishes, 91: 165-176.

Brooks, K., Rowart, D., Pierce, S. J., Jouannet, D. \& Vely, M. (2010). Seeing spots: photo identification as a regional tool for Whale Shark identification. Western Indian Ocean, Journal of Marine Science, 9: 185-194.

Canese, S., Cardinali, A., Romeo, T., Giusti, M., Salvati, E., Angiolillo, M. \& Greco, S. (2011). Diving behavior of the giant devil ray in the Mediterranean Sea. Endangered Species Research, 14: 171-176.

Cárdenas-Palomo, N., Herrera-Silveira, J. \& Reyes, O. (2010). Distribución espacio-temporal de variables fisicoquímicas y biológicas en el hábitat del tiburón ballena Rhincodon typus (Orectolobiformes: Rhincodontidae) al norte del Caribe Mexicano. Revista de Biología Tropical, 58: 399-412.

Cisneros-Montemayor, A. M., Barnes-Mauthe, M., AlAbdulrazzak, D., Navarro-Holm, E., Sumaila, U. R. (2013). Global economic value of shark ecotourism: implications for conservation. Oryx. doi:10.1017/ S0030605312001718.

Clark, T. (2010). Abundance, home range, and movement patterns of manta rays (Manta alfredi, M. birostris) in Hawaii (Doctoral dissertation). University of Hawaii, Hawaii, USA.

Clua, E., Burray, N., Legendre, P., Mourier, J. \& Planes, S. (2011). Business partner or simple catch? The economic value of the sicklefin lemon shark in French Polynesia. Marine and Freshwater Research, 62: 764-770.

Colman, J. G. (1997). A review of the biology and ecology of the whale shark. Journal of Fish Biology, 51: 1219-1234.

Couturier, L. I., Jaine, F. R. A., Townsend, K. A., Weeks, S.J., Richardson, A. J. \& Bennett, M. (2011). Distribution, site affinity and regional movements of the manta ray, Manta alfredi (Krefft, 1868), along the east coast of Australia. Marine and Freshwater Research, 62: 628-637.

Deakos, M. H. (2010). Ecology and social behavior of a resident manta ray (Manta alfredi) population off Maui, Hawaii (Doctoral dissertation). University of Hawaii, Hawaii, USA.

Deakos, M. H., Bajer, J. D. \& Bedjer, L. (2011). Characteristics of a manta ray Manta alfredi population off Maui, Hawaii, and implications for management. Marine Ecology Progress Series, 429: 245-260.

de la Parra-Venegas, R., Hueter, R., González-Cano, J., Tyminski, J., Gregorio-Remolina, J., Maslanka, M., Ormos, A., Weigt, L., Carlson, B. \& Dove, A. (2011). 
An unprecedented aggregation of Whale sharks, Rhincodon typus, in Mexican coastal waters of the Caribbean sea. PLoS ONE 6: e18994.

de Lacy, T. \& Whitmore, M. (2006). Tourism and recreation. In M. Lockwood, G. L. Worboys \& A. Kothari (Eds.), Managing protected areas: a global guide (pp. 497-527). Earthscan Publications LTDA. London, United Kingdom.

Dewar, H., Mous, P., Domeier, M., Muljadi, A., Pet, J. \& Whitty, J. (2008). Movements and site fidelity of the giant manta ray, Manta birostris, in the Komodo Marine Park, Indonesia. Marine Biology, 155(2): 121-133

Díaz, J. M., Pinzón, J. H., Perdomo, A. M., Barrios, L. M. \& López-Victoria, M. (2001). Generalidades. In L. M. Barrios \& M. López-Victoria (Eds.), Gorgona Marina: Contribución al conocimiento de una isla única (pp. 17-27). INVEMAR, serie publicaciones especiales No 7, Santa Marta, Colombia.

Dudgeon, C. L., Noad, M. J. \& Lanyon, J. M. (2008). Abundance and demography of a seasonal aggregation of zebra sharks, Stegostoma fasciatum. Marine Ecology Progress Series, 368: 269-281.

Eckert, S. A. \& Stewart, B. S. (2001). Telemetry and satellite tracking of whale sharks, Rhincodon typus, in the Sea of Cortez, Mexico, and the North Pacific Ocean. Environmental Biology of Fishes, 60: 299-308.

Enríquez-Andrade, R. R., Rodríguez-Dowdell, N., ZavalaGonzález, A., Cárdenas-Torres, N., Vázquez-Haikin, A. \& Godínez-Reyes, C. (2003). Conservación y aprovechamiento sustentable del tiburón ballena a través del ecoturismo en Bahía de los Ángeles, Baja California. Universidad Autónoma Baja California, Ensenada, México.

Escarria, E., Beltrán-León, B. S., Giraldo, A. \& Zapata, F. (2007). Ichthyoplankton in the Nacional Natural Park Isla Gorgona (Pacific Ocean of Colombia) during September 2005. Investigaciones Marinas, 35: 127-133.

Freedman, R. \& Roy, S. S. (2012). Spatial patterning of Manta birostris in United States east coast offshore habitat. Applied Geography, 32: 652-659.

Gallagher, A. \& Hammerschlag, N. (2011). Global shark currency: the distribution, frequency and economic value of shark ecotourism. Current Issues in Tourism, 1: $1-6$.

Giraldo, A., Rodríguez-Rubio, E. \& Zapata, F. (2008). Condiciones oceanográficas en isla Gorgona, Pacífico oriental tropical de Colombia. Latin American Journal of Aquatic Research, 36: 121-128.

Giraldo, A. \& Zapata, F. A. (2006). Condiciones oceanográficas y biomasa zooplanctónica en la zona de influencia costera de isla Gorgona: agosto 2005-febrero 2006. (Informe Técnico Final). Proyecto de Investigación "Factores físicos, entorno oceanográfico y distribución de arrecifes coralinos en el PNN isla Gorgona». Universidad del Valle, Cali, Colombia.

Graham, R. T. \& Roberts, C. M. (2007). Assessing the size, growth rate and structure of a seasonal population of whale sharks (Rhincodon typus Smith 1828) using conventional tagging and photo identification. Fisheries Research, 84: 71-80.

Hazin, F. H. V., Vaske-Junior, T., Oliveira, P. G., Macena, B. C. L. \& Carvalho, F. (2008). Occurrences of whale shark (Rhincodon typus Smith, 1828) in the Saint Peter and Saint Paul archipelago, Brazil. Brazilian Journal of Biology, 68: 385-389.

Heupel, M. R., Simpfendorfer, C. A. \& Fitzpatrick, R. (2010). Large-scale movement and reef fidelity of grey reef sharks. PLoS ONE 5: e9650.

Heyman,W. D., Graham, R. T., Kjerfve, B. \& Johannes, R. E. (2001). Whale sharks Rhincodon typus aggregate to feed on fish spawn in Belize. Marine Ecology Progress Series, 215: 275-282.

Higginbottom, K. \& Scott, N. (2008). Strategic planning of wildlife tourism in Australia. Journal of Ecotourism, 7: 102-115.

Hleap, J. S., Lozano, R. A., Navia, A. F. \& Payán, L. F. (2010). Elasmobranquios del Parque Nacional Natural Gorgona. Ponencia realizada en II Encuentro colombiano sobre condrictios. Cali, Colombia.

Holmberg, J., Norman, B. \& Arzoumanian, Z. (2008). Robust, comparable population metrics through collaborative photo-monitoring of whale sharks, Rhincodon typus. Journal of Applied Ecology, 18: 222-233.

Holmberg, J., Norman B. \& Arzoumanian, Z. (2009). Estimating population size, structure, and residency time for whale sharks Rhincodon typus through collaborative photo-identification. Endangered Species Research, 7: 39-53.

Hussey, N. E., Stroh, N., Klaus, R., Chekchak, T. \& Kessel. S. T. (2013). SCUBA diver observations and placard tags to monitor grey reef sharks, Carcharhinus amblyrhynchos, at Sha'ab Rumi, The Sudan: assessment and future directions. Journal of the Marine Biological Association of the United Kingdom, 93: 299-308.

Huveneers, C., Luo, K., Otway, N. M. \& Harcourt, R. G. (2009). Assessing the distribution and relative abundance of wobbegong sharks (Orectolobidae) in New South Wales, Australia, using recreational scuba divers. Aquatic Living Resources, 22: 255-264.

Luiz, O. J., Balboni, A. P., Kodja, G., Andrade, M. \& Marum, H. (2009). Seasonal occurrences of Manta birostris (Chondrichthyes: Mobulidae) in southeastern Brazil. Ichthyological Research, 56: 96-99.

Marshall, A. D., Compagno, L. J. V. \& Bennett, M. B. (2009). Redescription of the genus Manta with 
resurrection of Manta alfredi (Krefft, 1868) (Chondrichthyes; Myliobatoidei; Mobulidae). Zootaxa, 2301: 1-28.

Marshall, A. D., Dudgeon, C. L. \& Bennett, M. B. (2011). Size and structure of a photographically identified population of manta rays Manta alfredi in southern Mozambique. Marine Biology, 158: 1111-1124.

Meekan, M. G., Bradshaw, C. J. A., Press, M., McLean, C., Richards, A., Quasnichka, S. \& Taylor, J. G. (2006). Population size and structure of whale sharks Rhincodon typus at Ningaloo Reef, Western Australia. Marine Ecology Progress Series, 319: 275-285.

Mejía-Falla, P. A., Navia, A. F., Tobón, A. \& Lozano, R. (2004). Frecuencia de avistamiento de tiburones y rayas en las costas colombianas. In Memorias I Simposium Nacional de Tiburones y Rayas (pp. 23-24). Universidad Nacional Autónoma de México, México.

Mejía-Falla, P. A., Caicedo-Pantoja, J. A., Tobón-López, A., Narváez, K., Lozano, R. \& Navia, A. F. (2007). Dinámica poblacional y uso de hábitat del tiburón aletiblanco (Triaenodon obesus), del tiburón ballena (Rhincodon typus) y de la mantarraya (Manta birostris) en el Parque Nacional Natural Gorgona, Pacífico colombiano. In Memorias del IX Simposio Colombiano de Ictiología. Universidad del Magdalena, Santa Marta, Colombia.

Mejía-Falla, P. A., Tobón-López, A., Navia, A. F., Narváez, K. \& Lozano, R. A. (2010). Avistamiento de elasmobranquios en aguas colombianas. In P. A. Mejía-Falla, K. Narváez, J. Bohórquez, F. Osaer, V. Ramírez \& J. S. Hleap. (Eds.), Libro de resúmenes II Encuentro colombiano sobre condrictios (pp. 29). Fundación SQUALUS, Cali, Colombia.

Mejía-Falla, P. A. \& Navia, A. F. (2011). Relationship between body size and geographic range size of elasmobranchs from the Tropical Eastern Pacific: An initial approximation for their conservation. Ciencias Marinas, 37: 305-321.

Musick, J. A., Harbin, M. M. \& Compagno, L. J. V. (2004). Historical zoogeography of selachii. In J. C. Carrier, J. A. Musick \& M. R. Heithaus (Eds.), Biology of sharks and their relatives (pp. 33 -78). CRC Press, New York, USA.

Navia, A. F., Narváez, K., Caicedo, J., Mejía-Falla, P. A. \& Giraldo, A. (2005). Tiburones y rayas en las principales eco-regiones del océano Pacífico y mar Caribe de Colombia. In Escuela de Ciencias del Mar de la Pontificia Universidad Católica de Chile (Ed.), Memorias XI Congreso Latinoamericano de Ciencias del Mar (pp. 331-332), Viña del Mar, Chile.

Navia, A. F., Mejía-Falla, P. A., Zapata, L. A., Bessudo, S., Soler, G. \& Rubio, E. A. (2009). Estado del conocimiento de tiburones y rayas del Pacífico Colombiano. In V. Puentes, A. F. Navia, P. A. Mejía-Falla, J. P.
Caldas, M. C. Diazgranados \& L. A. Zapata. (Eds.), Avances en el conocimiento de tiburones, rayas y quimeras de Colombia (pp. 133-194). Fundación SQUALUS, Ministerio de Ambiente Vivienda y Desarrollo Territorial, Instituto Colombiano Agropecuario, COLCIENCIAS, Conservación Internacional, WWF. Bogotá, Colombia.

Randall, J. E. (1977). Contribution to the biology of the whitetip reef shark (Triaenodon obesus). Pacific Science, 31: 143-164.

Robbins, W. D. (2006). Abundance, demography and population structure of the grey reef shark (Carcharhinus amblyrhynchos) and the whitetip reef shark (Triaenodon obesus) (Fam. Carcharhinidae) (Doctoral dissertation). James Cook University, Australia.

Shaller, P. (2006). Husbandry and reproduction of Whitetip reef sharks Triaenodon obesus at Steinhart Aquarium, San Francisco. International Zoo Yearbook, 40: $232-240$.

Speed, C. W., Meekan, M. G., Field, I. C., McMahon, C. R., Stevens, J. D., McGregor, F., Huveneers, C., Berger, Y. \& Bradshaw, C. J. A. (2011). Spatial and temporal movement patterns of a multi-species coastal reef shark aggregation. Marine Ecology Progress Series, 429: 261-275.

Stacey, J., Karam, J., Dwayer, D., Speed, C. \& Meekan, M. (2008). Assessing traditional ecological knowledge of Whale Sharks (Rhincodon typus) in eastern Indonesia: A pilot study with fishing communities in Nusa Tenggara Timur (Technical Report). School for Environmental Research, Charles Darwin University, Australia.

Theberge, M. M. \& Dearden, P. (2006). Detecting a decline in whale sharks Rhincodon typus sightings in the Andaman Sea, Thailand using ecotourist operatorcollected data. Oryx, 40: 337-342.

Topelko, K. N. \& Dearden, P. (2005). The shark watching industry and its potential contribution to shark conservation. Journal of Ecotourism, 4: 108-128.

Ward-Paige, C. A. \& Lotze, H. K. (2011). Assessing the value of recreational divers for censusing elasmobranchs. PLoS ONE 6: e25609.

Wilson, S. G., Taylor, J. G. \& Pearce, A. F. (2001). The seasonal aggregation of whale sharks at Ningaloo Reef, Western Australia: currents, migrations and the El Nino/Southern Oscillation. Environmental Biology of Fishes, 61: 1-11.

Whitney, N. M., Pyle, R. L., Holland, K. N. \& Barcz, J. T. (2012). Movements, reproductive seasonality, and fisheries interactions in the whitetip reef shark (Triaenodon obesus) from community-contributed photographs. Environmental Biology of Fishes, 93: 121-136. 